\title{
REFLECTION OF OBLIQUE SHOCK WAVES IN INCOMPRESSIBLE ELASTIC SOLIDS
}

\author{
B. DUSZCZYK ${ }^{1}$, S. KOSINSKI $^{2}$ AND Z. WESOLOWSKI ${ }^{3}$
}

(Received 30 March 1983; revised 20 June 1984)

\begin{abstract}
Using a semi-inverse method proposed by Wright the reflection of a finite elastic plane shock wave at a plane boundary of a special elastic incompressible material is examined. Three types of boundary conditions are considered. In the case of frictionless-rigid boundary the reflected wave is a single simple wave. For clamped boundary the solution indicates a possibility of irregular reflection as well. There is no reflection solution in the case of a free boundary.
\end{abstract}

\section{Introduction}

The reflection of a finite plane shock wave at a plane boundary in nonlinear homogeneous simple elastic material was investigated by Wright $[6,7]$ and Duvaut [1]. Both authors obtained the solution in a form of centered shocks and simple waves, but the treatment and scope of these two presentations differ considerably.

Using a strictly mechanical theory, Wright presented a semi-inverse method of finding the reflected waves. In this method it is assumed that the incident shock, with the angle of incidence $\theta_{0}$ smaller than a certain critical angle $\theta_{c}$, is given $a$ priori, and that the medium ahead of the shock has a fixed state; this means that the state immediately behind the shock is also known. It is also assumed that the state behind the wave and the state at the boundary (compatible with the

\footnotetext{
${ }^{1}$ Department of Mathematics, University of Papua, New Guinea.

${ }^{2}$ Institute of Civil Engineering I-32, Polytechnical University, Lodz, Poland.

${ }^{3}$ Institute of Fundamental Technological Research, Polish Academy of Sciences, Warsaw, Poland.

(c) Copyright Australian Mathematical Society 1985, Serial-fee code 0334-2700/85
} 
boundary conditions) are connected by means of a sequence of one parameter families of reflected waves (simple waves), centered at the moving line of contact between the incident shock wave and the plane boundary. In general, three such families are required. The reflection problem then reduces to an initial-boundary value problem for a system of ordinary differential equations governing the variation of the deformation gradient and velocity fields in the regions of simple waves. Its solution determines the fan-shaped regions of simpie waves and the distribution and strength of the wavelets within each wave.

In some cases the assumed reflection pattern may fail the admissibility test; the pattern must be then modified to include shocks or a combination of shocks and simple waves. Both the shocks and the simple waves are given by one-parameter families of functions. A typical combination is a joint family of the reflected shocks and simple waves which may be connected to the same state. The transition from one type of the wave to the other one within this family can be obtained by continuous variation of the family parameter. Such a combination is called a composite wave [7].

In this paper a problem of reflection at a plane boundary of an oblique plane transverse shock wave propagating through an elastic rubber-like Zahorski's material [8] is examined. The material region ahead of the incident shock is assumed to be unstrained and at rest. The strength and the directions of propagation and polarisation of the incident shock are given; then the basic assumption is made that the reflection solution is in the form of a single simple wave centered on the line of incidence.

Section 2 contains a summary of necessary theory and derivation of the propagation condition for simple waves in incompressible materials. In Section 3 geometric and analytic descriptions of the incident shock and reflected simple wave are given; then, for a special isotropic incompressible material the shock propagation condition, the differential equations for the deformation gradient, velocity and the $p\left(X^{\alpha}\right)$ function in the region of simple wave are derived. Three types of boundary conditions and the corresponding initial-boundary value problems are considered in Section 4. Section 5 contains the numerical analysis of the reflection solution for clamped and frictionless-rigid boundary conditions. The results are illustrated graphically.

\section{Shocks and simple waves}

The motion of the continuum is given by $x^{i}=x^{i}\left(X^{\alpha}, t\right)$ where $x^{i}$ and $X^{\alpha}$ are the Cartesian coordinates of a material particle in the present configuration $B$ and the reference configuration $B_{R}$ respectively. The deformation gradient $x_{\alpha}^{i}$, its 
inverse $X_{t}^{\alpha}$ and the velocity are defined by

$$
x_{\alpha}^{i}=\frac{\partial x^{i}}{\partial X^{\alpha}}, \quad X_{1}^{\alpha}=\frac{\partial X^{\alpha}}{\partial x^{i}}, \quad \dot{x}^{\prime}=\frac{\partial x^{i}}{\partial t}=u^{i} .
$$

It is assumed that the material is homogeneous, elastic, and incompressible. The incompressibility constraint requires that

$$
J=\operatorname{det}\left(x_{\alpha}^{i}\right)=1
$$

The Piola-Kirchhoff stress tensor for such material is

$$
T_{R i}^{\alpha}=\rho_{R} \frac{\partial \sigma}{\partial x_{\alpha}^{\prime}}+p X_{i}^{\alpha}
$$

where $\sigma$ denotes internal energy per unit mass in $B_{R}, \rho_{R}=\rho$ is the density and $p=p\left(X^{\alpha}\right)$ is an arbitrary scalar function (hydrostatic pressure).

If the stress and velocity fields are differentiable, then the equations expressing balance of momentum and moment of momentum are

$$
T_{R i, \alpha}^{\alpha}=\rho \dot{u}_{i}, \quad x_{\alpha}^{i} T_{R}^{j \alpha}=x_{\alpha}^{i} T_{R}^{i \alpha} .
$$

If the functions $x^{i}\left(X^{\alpha}, t\right)$ are continuous everywhere but have discontinuous first derivatives on some propagating surface $S\left(X^{\alpha}, t\right)=0$, the equations (2.4) must be replaced by the jump conditions on this surface

$$
\begin{gathered}
{\left[T_{R i}{ }^{\alpha}\right] N_{\alpha}=-\rho U_{v}\left[u_{\imath}\right],} \\
{\left[x_{\alpha}^{i}\right]=a^{i} N_{\alpha}, \quad\left[u^{i}\right]=-a^{i} U_{v} .}
\end{gathered}
$$

Such a surface is called a shock wave. The vector $N_{\alpha}$ is a material unit normal to the wave, $U_{v}$ is the speed of propagation along $N_{\alpha}$ and $a^{t}$ is the amplitude vector of the jump. The bold square brackets indicate the jump in the quantity enclosed across $S$; thus

$$
[\cdot]=(\cdot)^{B}-(\cdot)^{F}
$$

where the letters $F$ and $B$ refer to the limit values taken in front and rear sides of $S$ respectively.

Simple waves [4], [7] are defined to be regions of space-time in which all field quantities are continuous functions of a single parameter, say, $\lambda=G\left(X^{\alpha}, t\right)$. Regions of constant $\lambda$ are propagating surfaces, called wavelets, with unit normal and normal velocity in $B_{R}$ given by

$$
N_{\alpha}(\lambda)=\frac{G_{, \alpha}}{|\nabla G|}, \quad U(\lambda)=\frac{\dot{G}}{|\nabla G|} .
$$


The equation of motion (2.4), and a compatibility condition in the region of simple wave are

$$
\begin{aligned}
\frac{\partial T_{R i}^{\alpha}}{\partial x_{\beta}^{j}} x_{\beta}^{\prime \prime} G_{, \alpha} & =\rho u_{i}^{\prime} \dot{G}, \\
x_{\beta}^{\prime j} \dot{G} & =u^{\prime J} G_{, \beta}
\end{aligned}
$$

where the prime indicates differentiation with respect to $\lambda$. If $\dot{\sigma} \neq \hat{0}$, equations (2.7) may be written as

$$
\begin{aligned}
& \left(Q_{1 \jmath}-\rho U^{2} \delta_{i \jmath}\right) u^{\prime \jmath}=0, \\
& U x_{\beta}^{\prime j}+u^{\prime \jmath} N_{\beta}=0
\end{aligned}
$$

where

$$
Q_{\imath \jmath}=\frac{\partial T_{R i}^{\alpha}}{\partial x_{\beta}^{j}} N_{\alpha} N_{\beta}
$$

is the acoustic tensor. For an incompressible material substitution of (2.3) into (2.8), and the indentity $X_{i, \alpha}^{\alpha}=0$, lead to the equation

$$
\left(\bar{Q}_{i j}-\rho U^{2} \delta_{i j}\right) u^{\prime j}-p_{, \alpha} X_{i}^{\alpha} U /|\nabla G|=0
$$

or, since in the region of simple wave $p_{, a}=p^{\prime}|\nabla G| N_{\alpha}$, to the equation

$$
\left(\tilde{Q}_{i j}-\rho U^{2} \delta_{i j}\right) u^{\prime j}-p^{\prime} U X_{i}^{\alpha} N_{\alpha}=0 .
$$

We denote here

$$
\tilde{Q}_{\imath j}=\rho \sigma_{i j}^{\alpha \beta} N_{\alpha} N_{\beta}, \quad \sigma_{i j}^{\alpha \beta}=\frac{\partial^{2} \sigma}{\partial x_{\alpha}^{i} \partial x_{\beta}^{i}} .
$$

From the incompressibility condition (2.2) we have $J_{, \beta}=X_{i}^{\alpha} x_{\alpha}^{\prime \prime} G_{, \beta}=0$. Using this equation, together with (2.8) and the relation (cf. [5] page 111):

$$
U n^{i}=u N_{\alpha} X_{i}^{\alpha}
$$

where $n^{\prime}$ is a unit normal and $u$ the speed of propagation of the wave in $B$, the scalar $p^{\prime}$ can be eliminated:

$$
\begin{aligned}
& \tilde{Q}_{\imath} u^{\prime \prime} n^{i}-\rho U^{2} u_{i}^{\prime} n^{\prime}-p^{\prime} u^{-1} U^{2}=0, \\
& p^{\prime}(\lambda)=u U^{-2} \tilde{Q}_{i j} u^{\prime \prime} n^{i} .
\end{aligned}
$$

to obtain the propagation condition for simple waves in incompressible materials

$$
\left(Q_{i j}^{*}-\rho U^{2} \delta_{i j}\right) u^{\prime j}=0 \text {. }
$$

The tensor

$$
Q_{i j}^{*}=\tilde{Q}_{i j}-\tilde{Q}_{k j} n^{k} n_{i}
$$

is called the reduced acoustic tensor. 


\section{Incident shock and reflection pattern}

The constraint of incompressibility restricts the propagating waves to transverse waves only. In general, the reflection problem may have no solution in terms of simple waves, as there are at most two possible families of reflected waves in such a case; this means that there are two free parameters, with three boundary conditions to be met. However, solutions may exist for some types of incompressible materials, with particular deformation and boundary conditions. In this paper we examine such special cases.

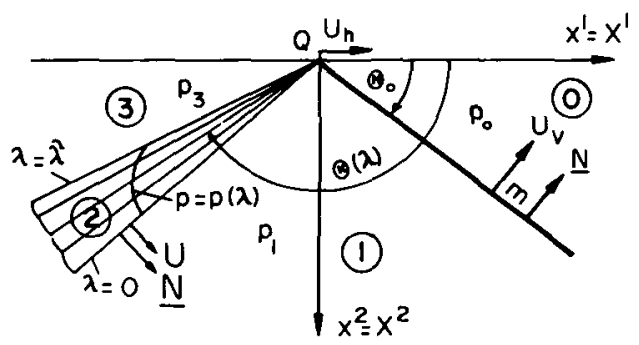

FIGURE 1. Incident shock and assumed reflection pattern.

Let $\left\{X^{\alpha}\right\}$ and $\left\{x^{i}\right\}$ be the Cartesian coordinates of an elastic particle in $B_{R}$ and $B$ respectively. Suppose that a plane shock wave propagates through an elastic incompressible material in the half space $X^{2} \geqslant 0$, and approaches its boundary $X^{2}=0$ at an angle $\theta_{0}$ (Figure 1), and the line of contact with the boundary is parallel to the $X^{3}$-axis. It is also assumed that the angle of incidence $\theta_{0}$ is smaller than a certain critical angle $\theta_{c}$ (refer (3.18)). Thus, this travelling discontinuity surface belongs to a one-parameter family of parallel planes, with normals

$$
N_{2}=\left(\sin \theta_{0},-\cos \theta_{0}, 0\right) .
$$

The reflection line (point $Q$ in Figure 1) moves along the boundary with speed $U_{h}=U_{v} / \sin \theta_{0}$ where $U_{v}$ is the shock speed. It is assumed that the reflected wave is a simple wave (region 2). The material region 0 in front of the incident shock is unstrained and at rest. Regions 1 and 3 have constant state.

Since all waves are centered on $Q$, we have for the reflected wave:

$$
\begin{gathered}
N_{\alpha}(\lambda)=(\sin \theta(\lambda),-\cos \theta(\lambda), 0), \\
U(\lambda)=U_{h} \sin \theta(\lambda),
\end{gathered}
$$

where $\theta(\lambda)$ is the angle of reflection and $\lambda$ is the reflected wave parameter. 
For (2.13) to have nonzero solution in $u^{\prime \prime}$ it is necessary that

$$
\begin{aligned}
\operatorname{det}\left(Q_{i j}^{*}-\rho U^{2} \delta_{i j}\right) & =(\sin \theta)^{6} \mathscr{P}(\tau)=0, \\
u^{\prime j} & =k r^{\prime} .
\end{aligned}
$$

$\mathscr{P}(\tau)$ is a sixth degree polynomial with real coefficients, $\tau=\cot \theta(\lambda)$, and $r^{i}$ is a right proper vector of $Q_{i j}^{*}$ corresponding to a particular proper value $\tau$. In (3.3) $k$ is an âtuitrary parameter; assuming $k=U$, it follows from (2.7b) and (3.3b) that:

$$
x_{\beta}^{n}=-r^{i} N_{\beta}, \quad u^{\prime \prime}=U r^{i} .
$$

These are the ordinary differential equations for the gradient $x_{\alpha}^{i}$ and velocity $u^{i}$ in the region of simple wave. They can be solved, with the initial conditions taken in region 1.

A detailed discussion and geometric interpretation of the roots of $\mathscr{P}(\tau)$ can be found in [7]. In this paper we shall only state that for a simple wave $\tau$ must be a real decreasing function of $\lambda$, when $\lambda$ changes from its initial value 0 to the extreme value $\tilde{\lambda}$ (which may be negative); it means that its wavelets (rays) diverge when $\lambda$ varies from 0 to $\tilde{\lambda}$. If $\tau(\lambda)$ increases, then the assumed reflection pattern should be modified to include shocks as well [7].

We assume that the incompressible elastic material that fills the region $X^{2} \geqslant 0$ (Figure 1) is isotropic, and it is defined by the constitutive equation

$$
W\left(I_{1}, I_{2}\right)=\rho \sigma\left(I_{1}, I_{2}\right)=C_{1}\left(I_{1}-3\right)+C_{2}\left(I_{2}-3\right)+C_{3}\left(I_{1}^{2}-9\right)
$$

proposed by Zahorski [8], where

$$
I_{1}=B_{i}^{i}, \quad I_{2}=\frac{1}{2}\left(B_{i}^{i} B_{j}^{j}-B_{j}^{i} B_{i}^{\prime}\right)
$$

are the invariants of the left Cauchy-Green strain tensor $B^{i j}$.

The sets of values for $C_{1}, C_{2}, C_{3}$ for three kinds of rubber are given in [9].

Furthermore, we assume that the direction of polarisation, given by unit vector $d^{i}$, is parallel to the $X^{3}$-axis:

$$
d^{i}=d_{1}=(0,0,1) .
$$

The jump conditions (2.5b) are now

$$
\left[\dot{x}^{i}\right]=-m U_{v} d^{\prime}, \quad\left[x_{\alpha}^{i}\right]=m d^{\prime} N_{\alpha}
$$

where $a^{i}=m d^{i}$, and $m$ is the shock strength. It is clear that only the jumps of two components of the deformation gradient and one of the velocity are not zero. Since the medium in front of the shock is unstrained and at rest, these jumps are

$$
\begin{gathered}
{\left[\dot{x}^{3}\right]=\left(\dot{x}^{3}\right)^{B}=-m U_{v},} \\
{\left[x_{1}^{3}\right]=\left(x_{1}^{3}\right)^{B}=m \sin \theta_{0},\left[x_{2}^{3}\right]=\left(x_{2}^{3}\right)^{B}=-m \cos \theta_{0} .}
\end{gathered}
$$


Substituting (3.5) and (3.8a) into (2.5a) we obtain the propagation condition

$$
U_{v}^{2}=\frac{2}{\rho}\left[C_{1}+C_{2}+6 C_{3}+2 C_{3} m^{2}\right] .
$$

This means that for Zahorski's material the squared speed of the shock wave is a quadratic function of the shock strength.

The material region behind the wave front should remain elastic. Hence, the discontinuity jumps cannot be arbitrary, and the appropriate estimates for $m$ should be established. In the further numerical analysis the jump estimates presented in [2] for the material (3.5) will be used.

Since by (3.8a) $\left(x_{\alpha}^{i}\right)^{B}-\delta_{\alpha}^{i}=m d^{i} N_{\alpha}=\hat{x}_{\alpha}^{i}$, the deformation gradient and its inverse in region 1 are

$$
\left(x_{\alpha}^{i}\right)^{B}=\left[\begin{array}{ccc}
1 & 0 & 0 \\
0 & 1 & 0 \\
\hat{x}^{3}{ }_{1} & \hat{x}^{3}{ }_{2} & 1
\end{array}\right], \quad\left(X_{i}^{\alpha}\right)^{B}=\left[\begin{array}{ccc}
1 & 0 & 0 \\
0 & 1 & 0 \\
-\hat{x}^{3}{ }_{1} & -\hat{x}^{3}{ }_{2} & 1
\end{array}\right] .
$$

The components of $T_{R i}^{\alpha}$ and the elasticities $\sigma_{i j}^{\alpha \beta}$ (refer (2.10)) required in this paper are then evaluated in region 1 :

$$
\begin{array}{ll}
T_{R 1}{ }^{1}=2 \rho\left\{\sigma_{1}+\sigma_{2}\left[2+\left(\hat{x}^{3}\right)^{2}\right]\right\}+p, & T_{R 1}{ }^{3}=-2 \rho \sigma_{2} \hat{x}^{3}-p \hat{x}^{3}, \\
T_{R 2}{ }^{2}=2 \rho\left\{\sigma_{1}+\sigma_{2}\left[2+\left(\hat{x}^{3}\right)^{2}\right]\right\}+p, & T_{R 3}{ }^{1}=2 \rho\left(\sigma_{1}+\sigma_{2}\right) \hat{x}^{3}{ }_{1}, \\
T_{R 3}{ }^{3}=2 \rho\left\{\sigma_{1}+2 \sigma_{2}\right\}+p, & T_{R 2}{ }^{3}=-2 \rho \sigma_{2} \hat{x}^{3}{ }_{2}-p \hat{x}^{3}{ }_{2}, \\
T_{R 1}{ }^{2}=T_{R 2}{ }^{2}=-2 \rho \sigma_{2} \hat{x}^{3} \hat{x}^{3}, & T_{R 3}{ }^{2}=2 \rho\left(\sigma_{1}+\sigma_{2}\right) \hat{x}^{3}{ }_{2} . \\
\sigma_{33}^{11}=2\left(\sigma_{1}+\sigma_{2}\right)+4 \sigma_{11}\left(\hat{x}^{3}\right)^{2}, & \sigma_{33}^{22}=2\left(\sigma_{1}+\sigma_{2}\right)+4 \sigma_{11}\left(\hat{x}^{3}{ }_{2}\right)^{2}, \\
\sigma_{33}^{12}=\sigma_{33}^{21}=4 \sigma_{11} \hat{x}^{3} \hat{x}^{3}{ }_{2} . &
\end{array}
$$

where

$$
\begin{gathered}
\sigma_{1}=\frac{\partial \sigma}{\partial I_{1}}=\frac{1}{\rho}\left(C_{1}+2 C_{3} I_{1}\right), \quad \sigma_{2}=\frac{\partial \sigma}{\partial I_{2}}=\frac{1}{\rho} C_{2}, \\
\sigma_{11}=\frac{\partial^{2} \sigma}{\partial I_{1}^{2}}=\frac{2}{\rho} C_{3}, \quad I_{1}=I_{2}=3+\left(\hat{x}_{1}^{3}\right)^{2}+\left(\hat{x}_{2}{ }^{3}\right)^{2} .
\end{gathered}
$$

From (3.7) and (3.8) it follows that

$$
u^{i}=\left(0,0, u^{3}\right), \quad u^{3}=-m U_{v} .
$$

This implies that all motion is restricted to the $x^{3}$ direction.

By (3.11) and the assumption that $u^{3}$ is not zero the condition (2.13) is reduced to a single equation (refer (3.3));

$$
Q_{33}^{*}-\rho U^{2}=0 .
$$


From (2.11), (3.2) and (3.10) it follows that $n_{3}=0$ and, by (2.10) and (2.14), $Q_{33}^{*}=\tilde{Q}_{33}=\rho \sigma_{33}^{\alpha \beta} N_{\alpha} N_{\beta}$. Denoting

$$
\hat{N}_{\alpha}=N_{\alpha} / \sin \theta=(1,-\tau, 0), \quad \tau(\lambda)=\cot \theta(\lambda),
$$

and using (3.2b), we can rewrite (3.14) as a quadratic equation in $\tau$ :

$$
\begin{aligned}
& \sigma_{1 j}^{\alpha \beta} N_{\alpha} N_{\beta}-U_{h}^{2}=0, \\
& \sigma_{33}^{22} \tau^{2}-2 \sigma_{33}^{12} \tau+\left(\sigma_{33}^{11}-U_{n}^{2}\right)=0 .
\end{aligned}
$$

Its smaller root $(c f .[6])$,

$$
\tau=\frac{\sigma_{33}^{12}}{\sigma_{33}^{22}}-\left[\left(\frac{\sigma_{33}^{12}}{\sigma_{33}^{22}}\right)^{2}-\frac{\sigma_{33}^{11}-U_{h}^{2}}{\sigma_{33}^{22}}\right]^{1 / 2}
$$

indicates the planes of reflected wavelets. The requirement that the roots are real gives the condition for the critical angle $\theta_{i}$ : it is the largest angle $\theta$ for which the following inequality holds

$$
(\sin \theta)^{-2} \geqslant U_{v}^{-2}\left[\sigma_{33}^{11}-\left(\sigma_{33}^{12}\right)^{2} / \sigma_{33}^{22}\right] .
$$

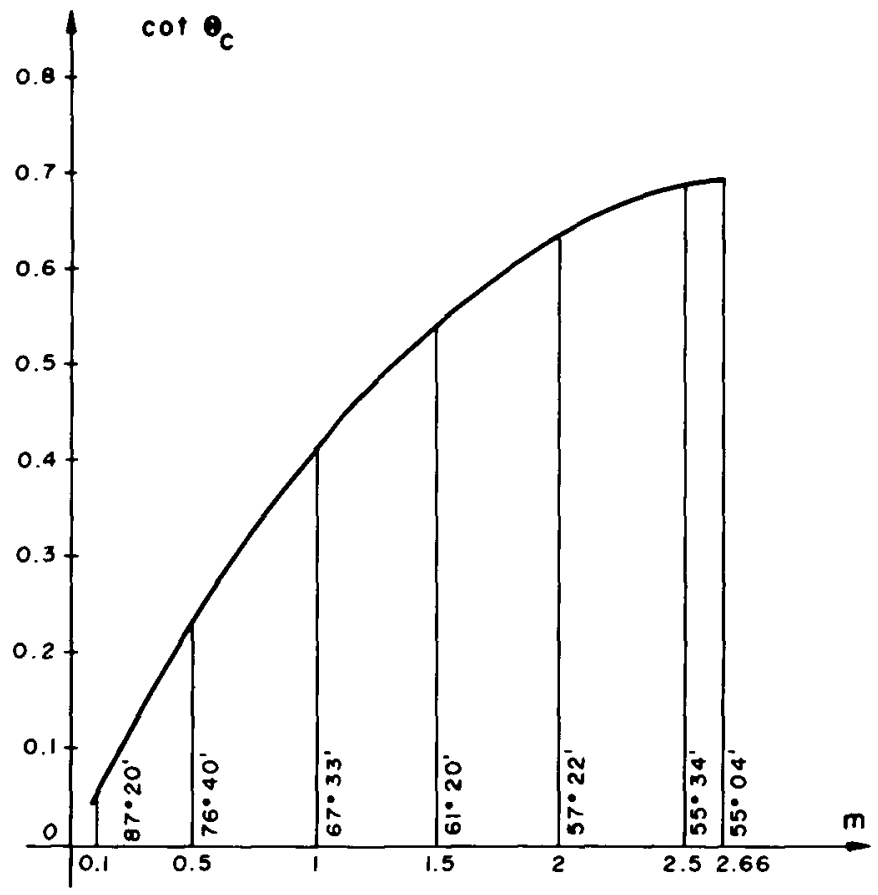

FIGURE 2. Relation between critical angle and shock strength. 


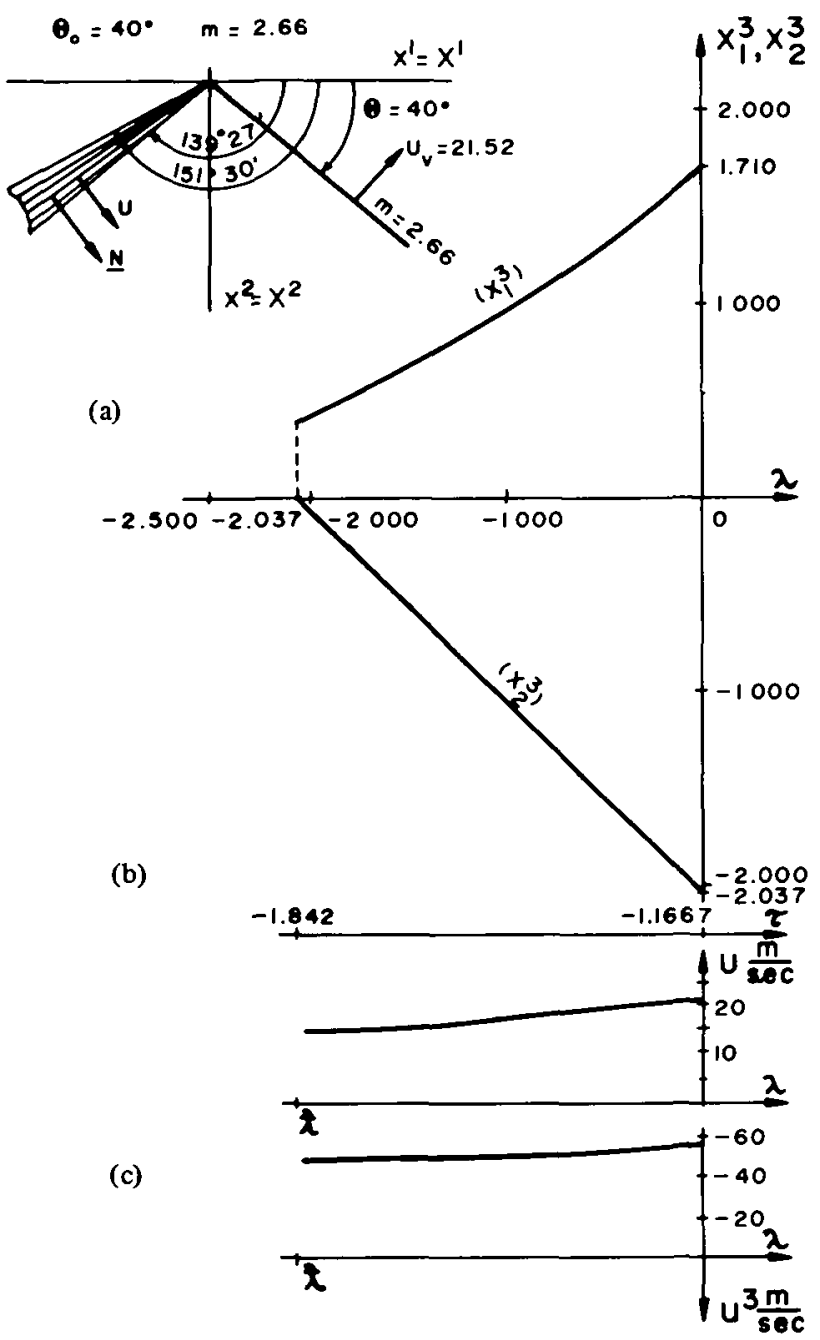

FIGURE 3. (a) Deformation gradient, (b) speed of propagation, (c) particle velocity in region of simple wave.

The elasticities $\sigma_{i j}^{\alpha \beta}$ are evaluated in region 1, with the material constants: $C_{1}=0.64, C_{2}=0.09, C_{3}=0.07$. (See [9], for rubber OKA-1; the constants are expressed in $\mathrm{kg} / \mathrm{cm}^{2}$.) Figure 2 shows that the critical angle $\theta_{c}$ decreases with increasing shock strength $m$.

Equation (2.12) was derived for arbitrary homogeneous incompressible elastic material and arbitrary deformation. In the special case considered here this equation is reduced to the form,

$$
p^{\prime}(\lambda)=U^{-1}\left(\tilde{Q}_{13} N_{1}+\tilde{Q}_{23} N_{2}\right) u^{\prime 3} .
$$




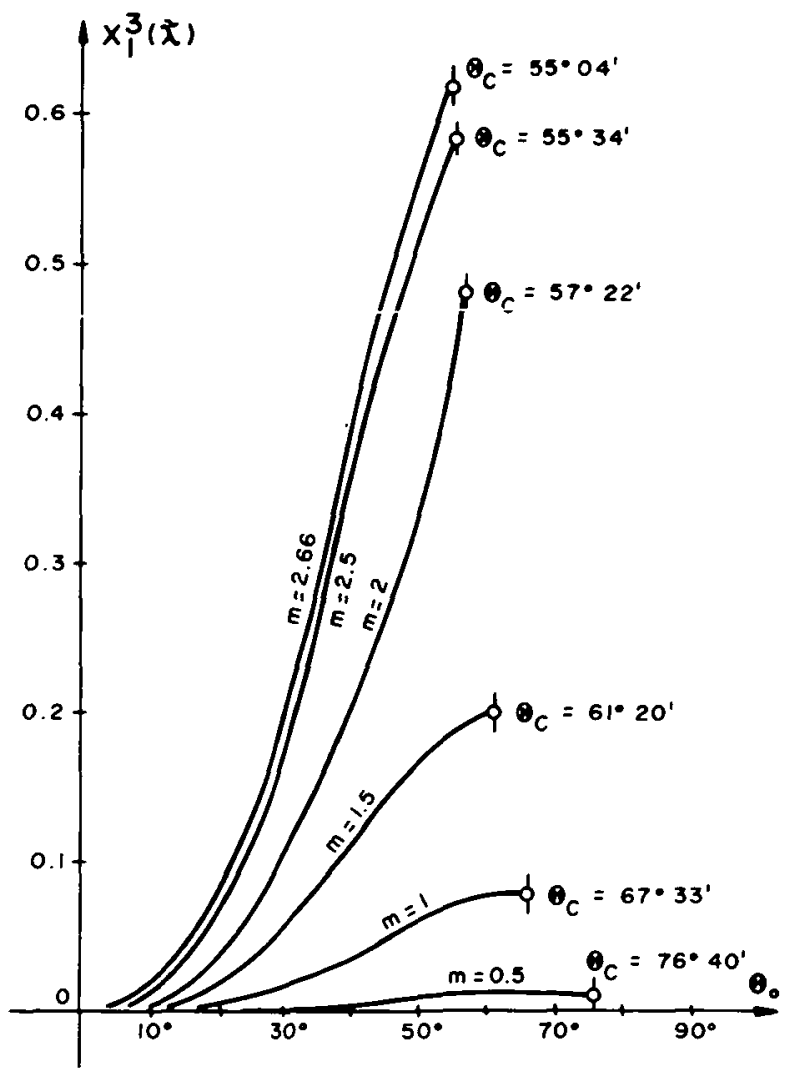

FIGURE 4. Relation between $\hat{x}^{3}{ }_{1}$ and $\theta_{0}$ on the trailing edge of simple wave.

Substitution of (3.10), (3.12) and (3.15) gives a differential equation for $p(\lambda)$ in terms of the deformation gradient and velocity fields in the region of simple wave,

$$
p^{\prime}(\lambda)=8 C_{3} U^{-1} u^{\prime 3}(1+\tau)^{-1 / 2}\left(\hat{x}_{1}^{3}-\tau \hat{x}^{3}{ }_{2}\right) \text {. }
$$

The problem of determining these two fields and the interval for the wave parameter $\lambda$ will be discussed in the next sections.

\section{Reflection calculations}

The simple wave is completely described by a one-parameter set of functions given by the ordinary differential equations (3.4), and satisfying given initial and boundary conditions. Equations (3.4) now are

$$
u^{\prime 3}=U_{n} f, \quad \hat{x}^{\prime 3}{ }_{1}=-f, \quad \hat{x}^{\prime 3}{ }_{2}=\tau f
$$


(a)

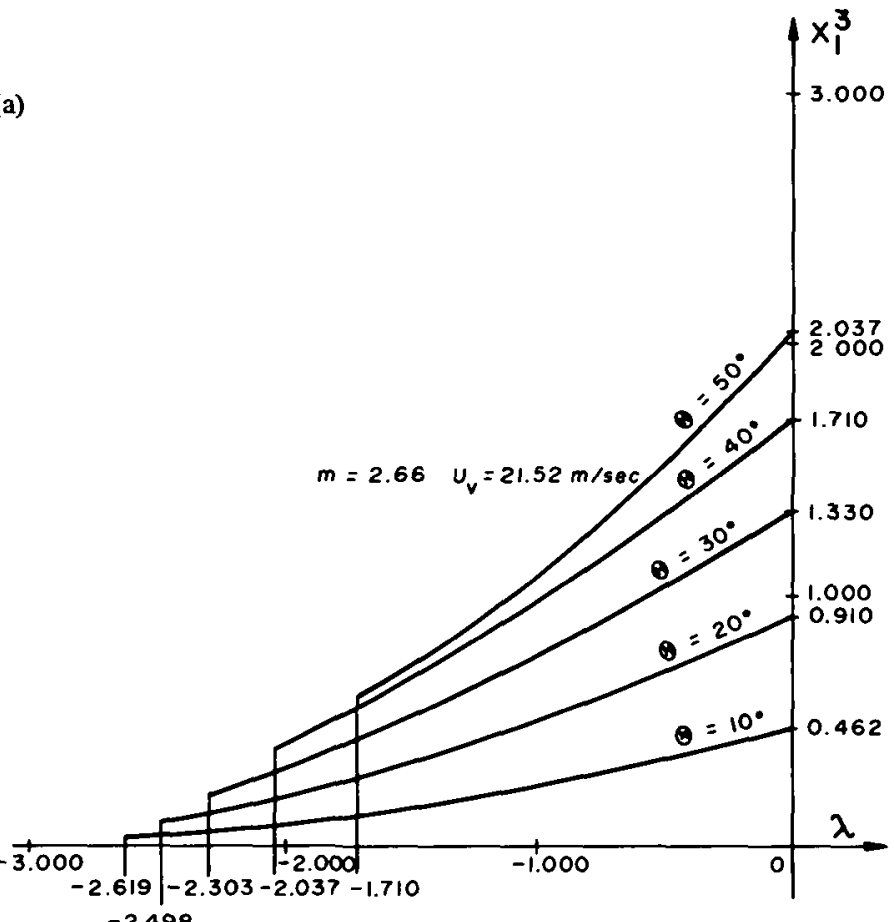
(b)

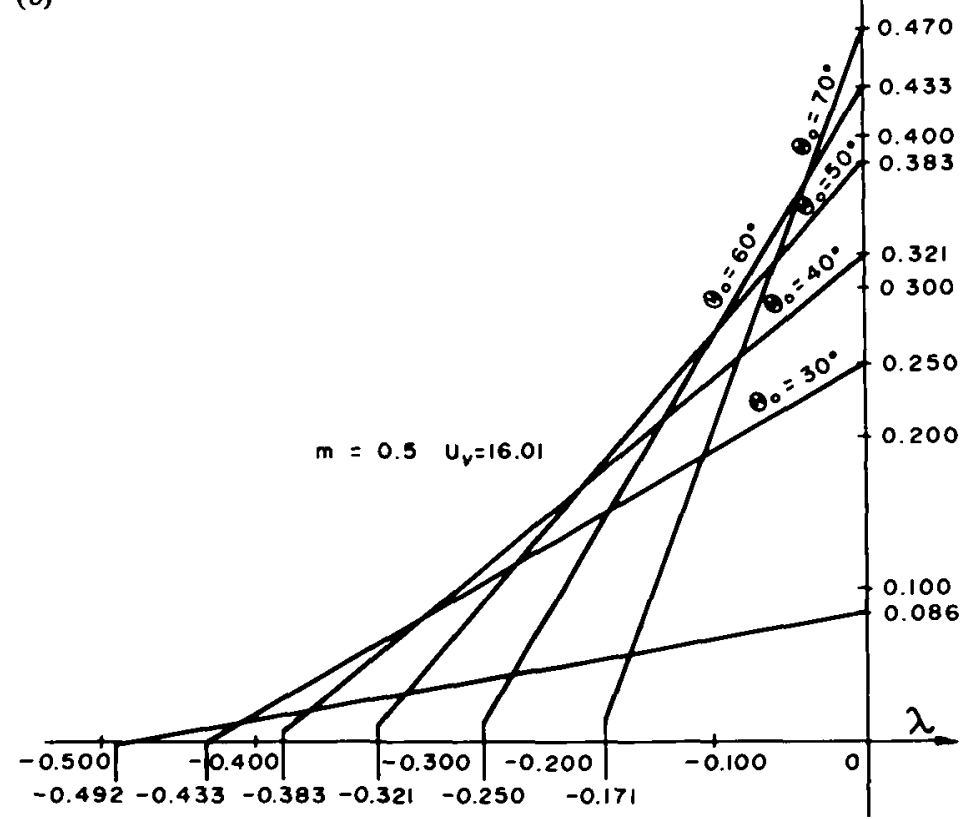

FIGURE 5. $\dot{x}^{3}{ }_{1}$ in region of simple wave (a) for $m=2.66$, (b) for $m=0.5$, and some values of $\theta_{0}$. 
where $f$ is an arbitrary function of the wave parameter $\lambda$. The deformation gradient and velocity are assumed to be continuous throughout regions 1-3 (Figure 1). Thus, the initial values for the differential equations that describe region 2 are the constant values of region 1; the final values in region 2 (at the trailing edge of the wave) are the constant values of region 3 , derived from the boundary conditions on the plane $X^{2}=0$.

The components of the deformation gradienti anu velocily behind the shock are given by $(3.8 \mathrm{~b} ; \mathrm{c})$; hence, the initial conditions for equations (4.1) are

$$
u^{3}(0)=-m U_{v}, \quad \hat{x}^{3}(0)=m \sin \theta_{0}, \quad \hat{x}^{3}{ }_{2}(0)=-m \cos \theta_{0},
$$

where the shock speed $U_{v}$ is given by (3.9).

\section{Frictionless-rigid boundary.}

Let us consider a case of "mixed" boundary conditions on the plane $X^{2}=0$, when the normal displacement and the shearing stresses $T_{R 1}{ }^{2}, T_{R 3}{ }^{2}$ are zero. Since all motion is restricted to the $X^{3}$-axis direction, the displacement condition is satisfied identically. The stress conditions

$$
\begin{aligned}
& T_{R 1}{ }^{2}=-2 \rho \sigma_{2} \hat{x}_{1}^{3} \hat{x}_{2}^{3}=0, \\
& T_{R 3}{ }^{2}=2 \rho\left(\sigma_{1}+\sigma_{2}\right) \hat{x}^{3}{ }_{1}=0
\end{aligned}
$$

are met when

$$
\hat{x}_{2}^{3}=0 \text { on } X^{2}=0 .
$$

To satisfy this condition it is convenient to choose in (4.1), $f=-\tau^{-1}$ for then the system (4.1) becomes

$$
\hat{x}^{\prime 3}{ }_{1}=\tau^{-1}, \quad \hat{x}^{\prime 3}{ }_{2}=-1, \quad u^{\prime 3}=-U_{h} x^{\prime 3}
$$

integrating the last two equations, with the initial conditions (4.2), we obtain

$$
\hat{x}_{2}{ }_{2}=-\lambda-m \cos \theta_{0}, \quad u^{3}=-U_{h}\left(\hat{x}_{1}{ }_{1}-m \sin \theta_{0}\right)-m U_{v},
$$

and the condition (4.4) is met when

$$
\lambda_{\text {final }}=\tilde{\lambda}=-m \cos \theta_{0} .
$$

Substitution for $\hat{x}^{3}{ }_{2}$ in $\tau$ (refer (3.17)) in equation (4.5a) gives a nonlinear differential equation for $\hat{x}^{3}{ }_{1}$ in the form, $\hat{x}^{\prime 3}{ }_{1}=g\left(\hat{x}^{3}{ }_{1}, \lambda\right), \tilde{\lambda} \leqslant \lambda \leqslant 0$. The initial condition is equation (4.2b). If $\theta_{0} \leqslant \theta_{c}, g$ is a real single-valued smooth function of both variables, and the initial value problem has a unique continuous solution $\hat{x}^{3}{ }_{1}(\lambda)$. This problem, however, can be solved only numerically. Substitution for $\hat{x}^{3}{ }_{1}$ and $\hat{x}^{3}{ }_{2}$ in (3.17) completes the solution. The function $\tau(\theta(\lambda))$ defines a 
family of wavelets of the reflected wave, and $\tau(\theta(0))$ indicates the leading edge of this wave. The final value for $\lambda$ is given by $(4.6)$ and $\tau(\theta(\bar{\lambda}))$ indicates the trailing edge of the wave.

\section{Clamped boundary.}

Let us assume that the incident shock is reflected from a rigidly constrained boundary; this means that

$$
u^{3}=0 \text { on } X^{2}=0 .
$$

To meet this condition it is convenient to choose in (4.1), $f=-U_{h}^{-1}$, for then the system (4.1) becomes

$$
u^{\prime 3}=-1, \quad \hat{x}^{\prime 3}{ }_{1}=U_{h}^{-1}, \quad \hat{x}^{\prime 3}{ }_{2}=-\tau U_{h}^{-1} ;
$$

integrating the first two equations, with the initial conditions (4.2), we obtain

$$
u^{3}=-\lambda-m U_{v}, \quad \hat{x}_{1}^{3}=U_{h}^{-1} \lambda+m \sin \theta_{0}
$$

and the condition (4.7) is satisfied when

$$
\bar{\lambda}=-m U_{v} \text {. }
$$

For $\lambda=\tilde{\lambda} \hat{x}^{3}{ }_{1}$ also vanishes: $\hat{x}^{3}{ }_{1}(\tilde{\lambda})=-m U_{h}^{-1} U_{v}+m \sin \theta_{0}=0$. Substitution for $\hat{x}^{3}{ }_{1}$ and $\tau$ (given by (3.17)) in (4.8c) leads to a nonlinear differential equation for $\hat{x}^{3}{ }_{2}$ which can be solved only numerically. Substitution for $\hat{x}^{3}{ }_{1}$ and $\hat{x}^{3}{ }_{2}$ in (3.17) completes the solution.

\section{Free boundary.}

Consider a case in which the stress vector $t_{i}=T_{R i}{ }^{\alpha} K_{\alpha}, K_{\alpha}=(0,-1,0)$, vanishes on the plane $X^{2}=0$. This means that for $X^{2}=0$,

$$
\begin{aligned}
& T_{R 1}{ }^{2}=-2 \rho \sigma_{2} \hat{x}_{1}^{3} \hat{x}^{3}{ }_{2}=0, \\
& T_{R 3}{ }^{2}=2 \rho\left(\sigma_{1}+\sigma_{2}\right) \hat{x}^{3}{ }_{2}=0, \\
& T_{R 3}{ }^{2}=2 \rho\left(\sigma_{1}+\sigma_{2}\right) \hat{x}^{3}{ }_{2}=0, \\
& T_{R 2}{ }^{2}=2 \rho\left\{\sigma_{1}+\sigma_{2}\left[2+\left(\hat{x}^{3}\right)^{2}\right]\right\}+p=0 .
\end{aligned}
$$

The first two equations led to the condition (4.4). The third equation which must be satisfied on $X^{2}=0$ in both regions 0 and 3 determines the hydrostatic pressure $p$ in region 0 :

and in region 3:

$$
p_{0}=-2\left(C_{1}+2 C_{2}+6 C_{3}\right)
$$

$$
p_{3}=p_{0}-2\left(C_{2}+2 C_{3}\right)\left(\hat{x}^{3}{ }_{1}(\tilde{\lambda})\right)^{2}
$$

where the $\hat{x}_{\alpha}^{i}(\bar{\lambda})$ are the values of the deformation gradient at the trailing edge of region 2 . 
The function $p$ is continuous throughout regions $1-3$, but it suffers a jump across the shock surface that separates regions 0 and 1 . To find $p_{1}$ in region 1 we use jump conditions (2.5). The first two equations: $\left[T_{R i}{ }^{\alpha}\right] N_{\alpha}=0$, or equivalently $\left[T_{R 1}{ }^{1}\right]=\tau^{2}\left[T_{R 2}{ }^{2}\right]$, together with (3.11) and (4.2), give the jump of $p$,

$$
|p|=p_{1}-p_{0}=-4 C_{3} m^{2},
$$

and the function $\bar{p}_{1}$ in ic gegion 1 of coñustant state is

$$
p_{1}=p_{0}-4 C_{3} m^{3}
$$

for arbitrary value of the incident angle $\theta_{0}<\theta_{c}$.

In region 2 the deformation gradient and velocity are completely determined by (4.5) and (4.6) as continuous functions of the wave parameter in the interval $\langle\tilde{\lambda}, 0\rangle$. Equations (4.5) and (4.6) are consistent with the first two conditions (4.10). The differential equation (3.19), after substitution for $\hat{x}_{1}^{3}, \hat{x}^{3}{ }_{2}$ and $u^{3}$, determines the hydrostatic pressure $p(\lambda)$ up to a constant in the interval $\langle\tilde{\lambda}, 0\rangle$. Direct integration, with the aid of (4.5), gives

$$
p(\lambda)=-4 C_{3}\left[\left(\hat{x}_{1}{ }_{1}(\lambda)\right)^{2}+\left(\hat{x}_{2}^{3}(\lambda)\right)^{3}\right]+C .
$$

Due to continuity throughout regions $1-3$, the function $p(\lambda)$ must satisfy two conditions, $p(0)=p_{1}$ and $p(\tilde{\lambda})=p_{3}$ where $p_{1}$ and $p_{3}$ are given by (4.12) and (4.13). Hence

$$
p(\lambda)=-4 C_{3}\left[\left(\hat{x}_{1}^{3}(\lambda)\right)^{2}+\left(\hat{x}_{2}^{3}(\lambda)\right)^{2}\right]+p_{0} .
$$

The second condition leads to the equation

$$
-4 C_{3}\left(x^{3}{ }_{1}(\tilde{\lambda})\right)^{2}=-2\left(C_{3}+2 C_{3}\right)\left(x^{3}{ }_{1}(\tilde{\lambda})\right)^{2}
$$

which is satisfied only when $C_{2}=0$. By assumption, for the material (3.5) considered in this paper, $C_{2} \neq 0$. This implies that the reflection problem for the case of a free boundary may have no solution in the form of a single simple wave.

\section{Numerical solutions}

The reflection solutions discussed in Section 4 are examined numerically for the material (3.5) with constants $C_{1}=0.64, C_{2}=0.09, C_{3}=0.07$. The Runge-Kutta method is used to solve the initial value problems (4.5), (4.2) and (4.8), (4.2) for some values of the incident angle $\theta_{0}<\theta_{c}$, and the following values of the incident shock strength [2]; $m=0.1,0.5,1.0,1.5,2.0,2.5,2.66$. 
Frictionless-rigid boundary.

Some characteristic results are shown in Figures 3-6.

Equation (4.5a), $x^{\prime 3}{ }_{1}=\tau^{-1}=\tan \theta(\lambda)$ states that the tangent to the curve $\hat{x}^{3}{ }_{1}=\hat{x}^{3}{ }_{1}(\lambda)$ at $\lambda=\lambda_{1}, \tilde{\lambda} \leqslant \lambda_{1} \leqslant 0$, is parallel to the corresponding ray $\theta_{1}=\theta\left(\lambda_{1}\right)$ in region 2 (Figure 1), and that the leading ray is $\theta_{1}=\theta(0)$. The graphs in Figures 3,4 and 5 show that $\hat{x}^{3}{ }_{1}(\lambda)$ is a decreasing concave-up function of $\lambda$ when $\lambda$ changes from 0 to $\bar{\lambda}<0$; this indicates that the function $\theta(\lambda)$ increases when $\lambda$

$\theta_{0}=5^{\circ} m=2.66$
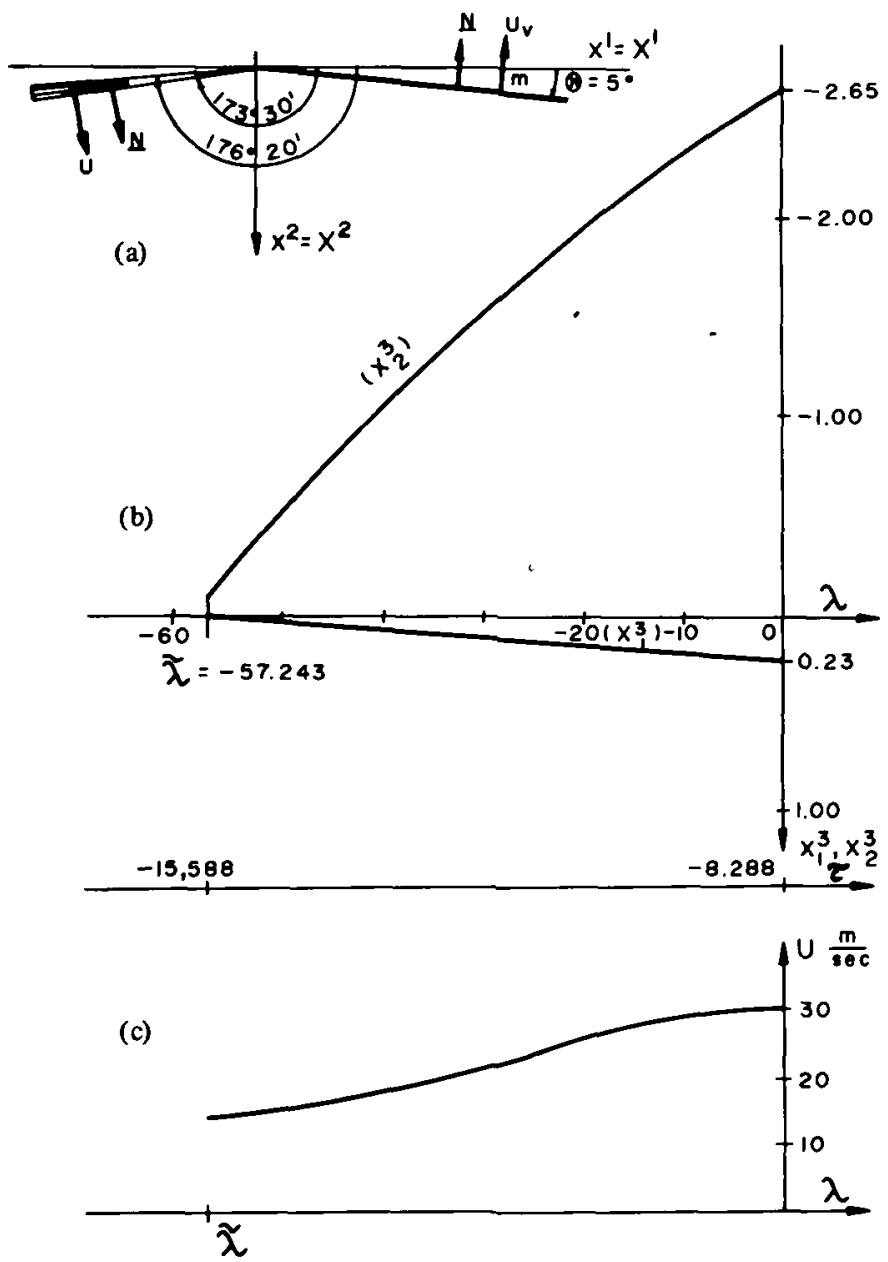

FIGURE 6. For $\theta_{0}=5^{\circ}, m=2.66$ (a) reflected simple wave, and (b) gradient. (c) wave speed as functions of $\lambda$. 


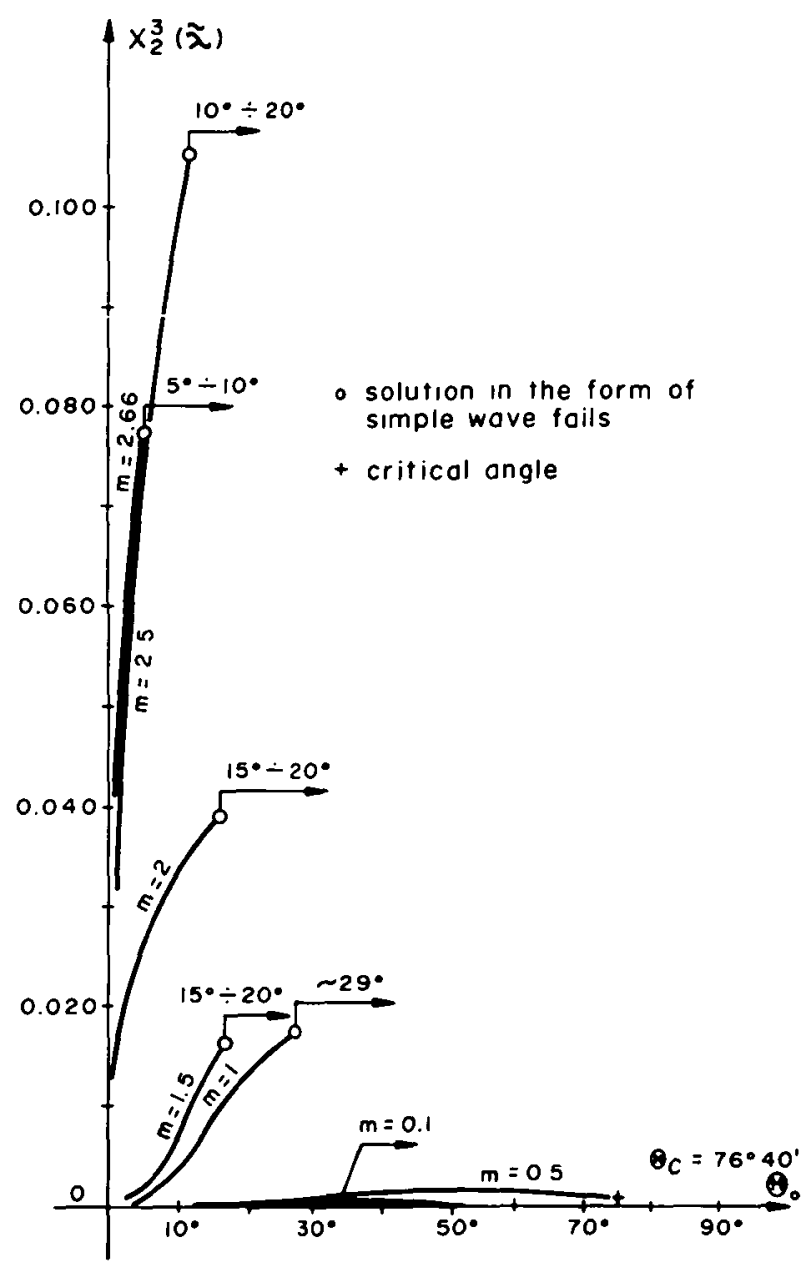

FIGURE 7. $\hat{x}_{2}{ }_{2}(\bar{\lambda})$ as function of incident angle and some values of $m$; indicated failure of reflection pattern.

changes from 0 to $\tilde{\lambda}$ and, according to the admissibility criterion, that region 2 is a reflected simple wave. The angle of this wedge shaped region is determined by the slopes of tangents at $\lambda=0$ and $\lambda=\tilde{\lambda}$. The width of the wave region, as measured by the angle of the wedge, increases with curvature of the curve $\hat{x}_{1}^{3}=\hat{x}_{1}^{3}(\lambda)$.

The numerical results show that the graphs of $\hat{x}^{3}{ }_{1}(\lambda)$ become less "curved" with the decreasing values of the incident shock parameters $\theta_{0}$ and $m$. For $m=0.5$ the curve $\hat{x}_{1}^{3}=\hat{x}_{1}^{3}(\lambda)$ (Figure $5(\mathrm{~b})$ ) is almost a straight line. In this case the reflected wave consists of a very "slim" fan of wavelets centered on $Q$. 


\section{Clamped boundary.}

Some results are shown in Figures 6 and 7.

It is characteristic for this case that the final value of the wave parameter is independent of $\theta_{0}$ (see (3.9) and (4.9)). The wavelet distributions within the wave however, depends on both parameters $\theta_{0}$ and $m$ (Figure 6(a)). The numerical analysis of the problem proves that only relatively weak shocks produce the reflected waves as simple waves for all $\theta_{0}<\theta_{c}$. For stronger shocks the pattern is preserved only for $\theta_{0}<\hat{\theta}_{0}$ where $\hat{\theta}_{0}$ is a certain specific angle depending on $m$, and $\hat{\theta}_{0}<\theta_{0}$; for $\theta_{0} \geqslant \hat{\theta}_{0}$ the wave function $\tau(\lambda)$ is no longer monotone in the whole interval $\langle\tilde{\lambda}, 0\rangle$. The assumed reflection pattern should be then modified to include a shock as well [6].

Figure 7 illustrates a situation where the solution fails the admissibility test on the trailing edge of the reflected simple wave. It can be seen that in each case (for $m \geqslant 1$ ) the reflected wave is a simple wave if $\theta_{0}$ is smaller than a certain specific angle $\hat{\theta}_{0}<\theta_{c}$; it is an indication that for $\theta_{0} \geqslant \hat{\theta}_{0}$ a shock could be formed at the trailing edge of the simple wave, and the reflected wave would be a shock-simple wave combination. It seems, however, that this combination could not be in a form of a composite wave centered on $Q$ : such a reflection pattern would not be stable [3] as the shock would propagate with sonic speed with respect to the medium ahead of it. In such a case rather an irregular reflection pattern, when the waves break away from $Q$ and interact with the incident shock, could be expected. The irregular reflections, however, are beyond the scope of this presentation and are not discussed here.

\section{References}

[1] G. Duvaut, "Phénomènes de réflexion, réfraction, intersection d'ondes planes uniformes dans des matériaux élastiques non linéaires", C. R. Acad. Sci. Paris Sér. A 264 (1967), 883-886.

[2] S. Kosinski, "Shock waves in selected nonlinear elastic materials", Ph.D. Thesis, Institute of Fundamental Technological Research, Warsaw, 1981 (in Polish).

[3] P. Lax, "Hyperbolic systems of conservation laws II", Comm. Pure Appl. Math. 10 (1957), 537-566.

[4] E. Varley, "Simple waves in general elastic material", Arch. Rational Mech. Anal. 20 (1965), 309-328.

[5] Z. Wesolowski, Dynamic problems in nonlinear elasticity (PWN-Publishers, Warsaw, 1974), (in Polish).

[6] T. W. Wright, "Reflection of oblique shock waves in elastic solids", Internat. J. Solids and Structures 7 (1971), 161-181.

[7] T. W. Wright, “Uniqueness of shock reflection patterns in elastic solids”, Arch. Rational Mech. Anal. 42 (1971), 115-127.

[8] S. Zahorski, "A form of the elastic potential for rubber-like materials", Arch. Mech. Stos. 5 (1959), 613-617.

[9] S. Zahorski, "Experimental investigation of certain mechanical properties of rubber", Rozprawy Inż 10 (1962), 193-207, (in Polish). 